\title{
Spectral Theory of Operator Pencils in the Hilbert Spaces
}

\author{
Rakhshanda Dzhabarzadeh \\ Department of functional analysis, Institute of Mathematics and Mechanics of NAS of Azerbaijan, Baku, Azerbaijan
}

Email address:

rakhshanda.dzhabarzade@rambler.ru (Dzhabarzadeh R. M.)

\section{To cite this article:}

Rakhshanda Dzhabarzadeh. Spectral Theory of Operator Pencils in the Hilbert Spaces. Pure and Applied Mathematics Journal. Special Issue: Spectral Theory of Multiparameter Operator Pencils and Its Applications. Vol. 4, No. 4-1, 2015, pp. 22-26.

doi: 10.11648/j.pamj.s.2015040401.15

\begin{abstract}
The theorem on possibility of multiple summation of the series on eigen and associated vectors of the operator pencil in the Hilbert space is proved. Research of multiple completeness and multiple expansions of eigen and associated vectors of such operator pencils are closely connected with the research of differential operator equation with the boundary conditions.
\end{abstract}

Keywords: Operator Pencil, Residue, Completely Continuous, Multiple

\section{Introduction}

Keldysh M.V. had proved the fundamental results [1] about the multiple completeness of the system of eigen and associated vectors (e.a) and properties of the eigen values for a wide class of polynomial pencils in the Hilbert space. This result allowed studying of boundary problems for partial differential equations and getting strong results for ordinary differential operator systems which can be applied to the study of equations of mathematical physics and differential equations with partial derivatives.

It is known that the general theory of equations

$$
\left(E-A_{0}-A_{1} \frac{d}{d t}-\ldots-A_{n} \frac{d^{n}}{d t^{n}}\right) x=0 .
$$

where $A_{i}(i=0,1, \ldots, n)$ - completely continuous operators, based on the theory of operator pencils

$$
L(\lambda)=A_{0}+\lambda A_{1} B+\ldots+\lambda^{n-1} A_{n-1} B^{n-1}+\lambda^{n} B^{n}
$$

Keldysh in [1] under the conditions that operators $A_{i}$ completely continuous; $B$ is completely continuous operator of finite order (series of the norms of eigenvalues in some positive order converges), $\operatorname{Ker} B=\{\vartheta\}$ proved the multiple completeness of the eigen and associated vectors of the operator pencil (1) in the Hilbert space. Later, this result was generalized by many authors in different directions. It should be noted of the works of J.E.Allahverdiev , M.G.Gasymov, A.G.Kostyuchenko, G.Radzievskii and others.
Theorems about multiple expansions of the root subspaces of the operator pencil $L(\lambda)$ are proved in the works of RM Dzhabarzadeh [2], V.N. Vizitey, A.S. Marcus [3] with the proviso that operators $A_{i} B^{-i}$ are bounded if $\frac{\lim }{k \rightarrow \infty} k \mu_{k}^{-p}=0$, and operators $A_{i} B^{-i}$ are completely continuous if $\varliminf_{k \rightarrow \infty} k \mu_{k}^{-p}<\infty$.

Through $\mu_{k}$ are designated the sequence of the characteristic values of the operator $L(\lambda)$ in order of increasing various modules taking into account their multiplicities.

In the work [6] it is proved the summation of the series on eigen and associated vectors of the operator pencil by the method of Abel. .

The work [5] is devoted also to the questions of multiple summation of series on eigen and associated vectors of operator pencil under the conditions that the resolvent of operator pencil on closed expanding indefinitely contours uniformly bounded .

Below it is presented a theorem asserting on multiple summation over the root subspaces of the operator pencil $L(\lambda)$, in other words, the possibility of $n$ - multiple expansions with brackets on eigen and associated vectors of the operator $L(\lambda)$. 


\section{Preliminary Definitions}

Let $A_{i}(i=0,1, \ldots, n), B$ in (1) be completely continuous linear operators, acting in a separable Hilbert space. The operator pencil (1) is known in the spectral theory of operators as a pencil of Keldysh.
Definition 1.Eigenvalue $\lambda$ of $L(\lambda)$ is called a complex number such that there exists a non-zero element $x \in H$ such that $L(\lambda) x=x$. This element $x \in H$ is called an eigenvector corresponding to the eigenvalue $L(\lambda)$.

Definition2. There is an associated vector to the eigenvector $x \in H$ if the following series of equations

$$
x_{i}=L(\lambda) x_{i}+\frac{1}{1 !} \frac{d}{d \lambda} L(\lambda) x_{i-1}+\ldots+\frac{1}{i !} \frac{d^{i}}{d \lambda^{i}} L(\lambda) x_{0} \quad i=1,2, \ldots k
$$

are satisfied.

Keldysh built the derivative systems according to the rule:

$$
x_{i}^{(j)}=\frac{d^{j}}{d t^{j}} e^{\lambda_{0} t}\left(x_{i}^{(0)}+x_{i-1}^{(0)} \frac{t}{1 !}+x_{i-2}^{(0)} \frac{t^{2}}{2 !}+\ldots+x_{0}^{(0)} \frac{t^{i}}{i !}\right)
$$

Definition3. [1] Under canonical system of e.a. vectors for eigenvalue $\lambda^{0}$ of the operator pencil (1) is understood the system

$$
z^{(k)}, z_{1}^{(k)}, \ldots, z_{p_{k}}^{(k)}(k=1,2, . .)
$$

possessing the following properties: elements $z^{(k)}(k=1,2, .$. form basis of a eigen subspace $M\left(\lambda^{0}\right)$; there is $z^{(1)}$ eigenvector which multiplicity reaches a possible maxima $p_{1}+1 ; z^{(k)}$ is eigenvector which is not expressing linearly though $z^{(1)}, \ldots, z^{(k-1)}$ which sum of multiplicities reaches a possible maxima $p_{k}+1$. Let's designate through $M\left(\lambda^{0}\right)$ linear span of eigen and associated vectors of the system (1), corresponding to an eigenvalue $\lambda^{0}$.

Linearly-independent elements (4) form a chain of eigen and associated vectors of (1). The multiplicity of eigenvector is equal to the greatest number of associated vectors to $z_{0, \ldots, 0}$ a plus 1 .

The sum $p_{1}+p_{2}+\ldots+p_{s}+s$ is a multiplicity of an eigenvalue $\lambda^{0}$.

Definition4. Under $n$-multiple completeness of eigen and associated vectors of the operator pencil $L(\lambda)$ in space $H$ is understood the possibility of approaching any $n$ element $f_{0}, f_{1}, \ldots, f_{n-1}$ of space $H$ by linear combinations of elements $\left\{x_{i}^{(j)}\right\}_{i=1}^{\infty}, j=0,1,2, \ldots, n-1$, respectively, with the same

$$
\bar{A}=\left(\begin{array}{cccc}
A_{0} & A_{1} & \ldots & A_{n-1} \\
0 & 0 & \ldots & 0 \\
. & . & \ldots & . \\
0 & 0 & \ldots & 0
\end{array}\right), \bar{B}=\left(\begin{array}{ccccc}
0 & 0 & 0 & \ldots & B \\
B & 0 & 0 & \ldots & 0 \\
0 & B & 0 & \ldots & 0 \\
. & . & . & \ldots & . \\
0 & 0 & 0 & \ldots B & 0
\end{array}\right), \bar{E}=\left(\begin{array}{cccc}
E & 0 & \ldots & 0 \\
0 & E & \ldots & 0 \\
. & . & \ldots & . \\
0 & 0 & \ldots & E
\end{array}\right) .
$$

Operator $\bar{A}$ is the completely continuous in the space $\bar{H}$ so the operators $A_{i},(i=0,1, \ldots, n-1)$ are completely continuous in the space $H$.

Operator $\bar{B}$ is a normal completely continuous , coefficients independent of the indices of the elements $f_{0}, f_{1}, \ldots, f_{n-1}$.

If at least for one point $\lambda=\lambda_{0}$ of the operator $L(\lambda)=I-A_{0}-\lambda A_{1} \ldots-\lambda^{n} A_{n} ;\left(A_{j} \in \sigma_{\infty}\right)$ invertible, then the set of eigen values of the pencil $L(\lambda)$ consists of isolated points of finite algebraic multiplicity [1].

\section{The Spectral Theorem on Multiple Summation of Series on Eigen and Associated Vectors of Operator Pencil}

The study of the spectral properties of the equation $L(\lambda) x=x$ in a Hilbert space $H$ is reduced to the study of the spectral properties of the equation $\bar{A} \bar{x}+\lambda \bar{B} \bar{x}=\bar{x}$ in the direct sum $\bar{H}$ of $n$ copies of the space $H$.

Indeed, $L(\lambda) x=x$ can be represented as a system of equations:

$$
\begin{aligned}
& x_{0}=A_{0} x_{0}+A_{1} x_{1}+\ldots+A_{n-1} x_{n-1}+\lambda B x_{n-1} \\
& x_{1}=\lambda B x_{0} \\
& x_{2}=\lambda B x_{1} \\
& \cdots \cdots \cdots \\
& x_{n-1}=\lambda B x_{n-2}
\end{aligned}
$$

Consider the equation

$$
\bar{A} \bar{x}+\lambda \bar{B} \bar{x}=\bar{x}
$$

where

characteristic values of $\bar{B}$ lie on $n$ rays emanated from origin, norms of characteristic values of operators $\bar{B}$ and $B$ coincide.

Theorem. Suppose that the following conditions are satisfied: 
a) $A_{i}$ and $B$ are complete continuous operators, $\left(E-A_{0}\right)^{-1}$ exists and bounded

b) there is a sequence of closed contours $\Gamma_{k}$ (circumferences) with radii $\rho\left(\Gamma_{k}\right) \rightarrow \infty$, such that for all $\lambda \in \Gamma_{k}$ we have

$$
\left\|(E-\lambda B)^{-1}\right\|<C
$$

Then we have the $n-$ multiple basis of eigen and associated vectors $L(\lambda)$ with brackets in the range of the operator $\left((\bar{E}-\bar{A})^{-1} \bar{B}\right)$. If $\overline{\left((\bar{E}-\bar{A})^{-1} \bar{B}\right)^{s+1}}=\bar{H}$ then the $n-$ multiple completeness of the system of eigen and associated vectors of the operator $L(\lambda)$ in the space $H$. takes place

Proof of Theorem.

We evaluate the resolvent - operator $(\bar{E}-\bar{A}-\lambda \bar{B})^{-1}$ for the values $\lambda \in \Gamma_{k}$. Let $\lambda \in \Gamma_{k}$ then.

$$
(\bar{E}-\bar{A}-\lambda \bar{B})^{-1}=(\bar{E}-\lambda \bar{B})^{-1}\left(\bar{E}-\bar{A}\left(\bar{E}-\lambda \bar{B}^{-1}\right)^{-1}\right.
$$

Suppose the estimate $\left\|(E-\lambda B)^{-1}\right\|<C$ holds for all $\lambda \in \Gamma_{k}$. The characteristic numbers of the operator $\bar{B}$ lie on the rays with the arguments $\frac{2 \pi k}{n}, k=1,2, \ldots, n$.The equalities

$$
\begin{gathered}
\left\|(\bar{E}-\lambda \bar{B})^{-1}\right\|=\max \left|\frac{1}{1-\mu_{k} e^{i \frac{2 \pi k}{n}} \lambda}\right|=\max \left|\frac{1}{1-\mu_{k}\left(e^{i \frac{2 \pi k}{n}} \lambda\right)}\right| . \\
e^{i \frac{2 \pi s}{n}} \lambda \in \Gamma_{k}, \text { if } \lambda \in \Gamma_{k},
\end{gathered}
$$

then accord to the condition of the theorem $\left\|(\bar{E}-\lambda \bar{B})^{-1}\right\|<C$

By condition b) of Theorem there exists a family of contours on which norms of the operators satisfy the conditions (6).

Further, $\left\|\bar{A}(\bar{E}-\lambda \bar{B})^{-1}\right\| \rightarrow 0$ and the norms of operator $\bar{A}(\bar{E}-\lambda \bar{B})^{-1}$ are closed to zero, starting with some $k$.(see[4],p309-310). Indeed, choose the arbitrary little number $\delta>0$ and represent operator $\bar{A}$ as sum of two operators $\bar{T}$ and $\bar{M}$, where $\bar{T}$ is finite dimensional operator and $\bar{M}$ is the operator arbitrary small norm.

$$
\bar{T}=\sum_{j=1}^{n}\left(\cdot, \psi_{j}\right) \phi_{j} \quad\left(\left\|\phi_{j}\right\|=1, j=1,2, \ldots, n\right) . \text { Let }\|\bar{M}\|<\frac{1}{2 C} \delta .
$$

Then for sufficient large meanings $\lambda$ we have

$$
\left\|\bar{M}(\bar{E}-\lambda \bar{B})^{-1}\right\|<\|\bar{M}\| C<\frac{1}{2 C} C \delta=\frac{1}{2} \delta, \quad \lambda \in \Gamma_{k}
$$

So the operator $\bar{B}$ is normal, completely continuous with the zero kernel, then

$$
(\bar{E}-\lambda \bar{B})^{-1}=\sum_{j=1}^{\infty} \frac{\mu_{j}(\cdot, \bar{e}) \overline{e_{j}}}{\mu_{j}-\lambda} \text {, where }\left\{\overline{e_{j}}\right\}_{1}^{\infty} \text { - complete }
$$

orthonormal sequence of eigen vectors of operator $\bar{B}$, and $\left\{\bar{\mu}_{j}\right\}_{1}^{\infty}$-corresponding system of characteristic values of the operator $\bar{B}$.

For sufficiently large $N$ and number $R(>)$

$$
\begin{aligned}
& \left(\sum_{j=N+1}^{\infty}\left\{\left(\bar{\psi}_{k}, \overline{e_{j}}\right)\right\}^{2}\right)^{\frac{1}{2}}<\frac{\delta C}{4 n} \quad(k=1,2, \ldots, n) \\
& \left(\sum_{j=1}^{N}\left|\frac{\mu_{j}}{\mu_{j}-\lambda}\right|^{2}\left|\left(\bar{\psi}_{k}, \bar{e}_{j}\right)\right|^{2}\right)^{\frac{1}{2}}<\frac{\delta}{4 \pi}
\end{aligned}
$$

Let $\bar{f}=\sum_{k=1}^{\infty}\left(\bar{f}, \overline{t_{k}}\right) e_{k}$ is the arbitrary element in $\bar{H}$.

Then

$$
\left\|\bar{T}(\bar{E}-\lambda \bar{B})^{-1} \bar{f}\right\| \leq\left[\sum_{k=1}^{n}\left(\sum_{j=1}^{\infty}\left|\frac{\mu_{j}}{\mu_{j}-\lambda}\left(\bar{\psi}_{k}, \bar{e}_{j}\right)\right|^{2}\left(\sum_{j=1}^{\infty}\left|\left(\bar{f}, \bar{e}_{j}\right)\right|\right)^{2}\right]^{\frac{1}{2}}\right.
$$

In virtue of

$$
\left\|(E-\lambda B)^{-1}\right\|=\max _{k}\left|\frac{\mu_{k}}{\mu_{k}-\lambda}\right|<C \quad \lambda \in \Gamma_{s}, s-1,2, \ldots
$$

and (8) we have

$$
\begin{aligned}
& \left\|\bar{A}(\bar{E}-\lambda \bar{B})^{-1}\right\| \leq\left\|\bar{T}(\bar{E}-\lambda \bar{B})^{-1}\right\|+\left\|\bar{M}(\bar{E}-\lambda \bar{B})^{-1}\right\|<q<1 \\
& \lambda>R, \quad \lambda \in \Gamma_{k}, k=1,2, \\
& \text { Put }\left\|\bar{A}(\bar{E}-\lambda \bar{B})^{-1}\right\|=q<1 \text { Series } \\
& \left(\bar{E}-\bar{A}\left(\bar{E}-\lambda \bar{B}^{-1}\right)^{-1}=1+\bar{A}(\bar{E}-\lambda \bar{B})^{-1}+\overline{\{A}(\bar{E}-\lambda \bar{B})^{-1}\right\}^{2}+\ldots
\end{aligned}
$$

converges and

$$
\left\|\left(\bar{E}-\bar{A}(\bar{E}-\lambda \bar{B})^{-1}\right)^{-1}\right\|<\frac{1}{1-q}
$$

By condition b) of Theorem there exists a family of contours on which norms of the operators $(\bar{E}-\bar{A}-\lambda \bar{B})^{-1}$ satisfy the conditions

$$
\left\|(\bar{E}-\bar{A}-\lambda \bar{B})^{-1}\right\| \leq \frac{1}{1-q}\left\|(\bar{E}-\lambda \bar{B})^{-1}\right\| \leq C_{1}
$$

We introduce the operator by the formula

$$
\bar{P}_{k} \bar{f}=\frac{1}{2 \pi i} \int_{\Gamma_{k}}\left(\bar{E}-\lambda(\bar{E}-\bar{A})^{-1} \bar{B}\right)^{-1} / \lambda^{2} \bar{f} d \lambda
$$


Introduce the domains $G_{k}$ on the complex plain, bounded by the contours $\Gamma_{k}$ and $\Gamma_{k-1}$. The contour, bounded the domain $G_{k}$, is denoted $\gamma_{k}$. It is known that the meaning of this integrand is equal to the sum of residues respectively of all poles of integrand inside of domain $G_{k}$. It is clear the contours may be chosen such manner to be satisfying the condition -of equality of sum all residues of integrand to zero.

Inside the domain $G_{k}$ bounded by the contour $\Gamma_{k}$ the integrand has the poles in characteristic numbers of the operator $\overline{(E}-\lambda(\bar{E}-\bar{A})^{-1} \bar{B}^{-1}$

The residue of integrand in the point $\lambda=0$ in domain $G_{1}$ is equal to $\left.\frac{d}{d \lambda} \frac{\left(\bar{E}-\lambda(\bar{E}-\bar{A})^{-1} \bar{B}\right)^{-1}}{\lambda^{2}} \bar{f}\right|_{\lambda=0}$. In the neighborhood of isolated characteristic value of operator $\overline{(E}-\lambda(\bar{E}-\bar{A})^{-1} \bar{B}^{-1}$ the general part of resolution of the operator of $\overline{(E}-\lambda(\bar{E}-\bar{A})^{-1} \bar{B}^{-1}$ has the form

$$
\sum_{k} \sum_{0 \leq s \leq m_{k}} \frac{\sum_{0 \leq i \leq s} \bar{x}_{i}^{(k)} \bar{z}_{s-i}^{(k)}}{\left(\lambda-\lambda_{i}\right)^{m_{k}-s+1}}
$$

$$
\text { In } \sum_{k} \sum_{0 \leq s \leq m_{k}} \frac{\sum_{0 \leq i \leq s} \bar{x}_{i}^{(k)} \bar{z}_{s-i}^{(k)}}{\left(\lambda-\lambda_{i}\right)^{m_{k}-s+1}}\left\{\bar{x}_{i}^{k}\right\}_{i=1}^{\infty} \text { is the canonical system of }
$$

eigen and associated vectors of the operator pencil $\bar{L}(\lambda)$, $\bar{z}_{s}^{(k)}$ is canonical system of eigen and associated system of

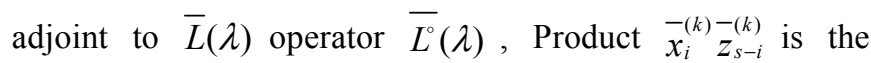
operator which on the element $\bar{f} \in \bar{H}$ is defined by the rule $\overline{\backslash C} \bar{f}=(\bar{f}, \bar{z}) \bar{y}$. In the isolated characteristic value $\lambda_{i}$ residue of integrand is $\frac{\overline{x_{0}(k)} \bar{z}_{m_{k}}+\ldots+\bar{x}_{m_{k}}^{(k)} z_{0}(k)}{\lambda_{i}^{2}}$.

Always we may choose the sequence of contours $\gamma_{k}$ for which $\sum_{k=1}^{\infty}\left\|P_{k+1}-P_{k}\right\|<\infty$, In this case the sum of all residues of the integrand respectively of all its poles is equal to zero.In particular, residue of integrand, respectively the point 0 is the element $\left((\bar{E}-\bar{A})^{-1} \bar{B}\right) \bar{f}$.

Indeed, so $\left\|(\bar{E}-\bar{A})^{-1} \bar{B}\right\|<1 \quad$ then the operator $\overline{(E}-\lambda(\bar{E}-\bar{A})^{-1} \bar{B}^{-1}$ in the small neighborhood of the point $\lambda=0$ is expanded in the converging series $\left(\bar{E}-\bar{A}\left(\bar{E}-\lambda \bar{B}^{-1}\right)^{-1}=1+\bar{A}(\bar{E}-\lambda \bar{B})^{-1}+\overline{\{A}(\bar{E}-\lambda \bar{B})^{-1}\right\}^{2}+\ldots$.

The following equalities

$$
\begin{gathered}
\left.\left.\overline{(E}-\lambda(\bar{E}-\bar{A})^{-1} \bar{B}\right)^{-1}=\bar{E}+\lambda(\bar{E}-\bar{A})^{-1} \bar{B}\right)+ \\
+\left(\lambda(\bar{E}-\bar{A})^{-1} \bar{B}^{2}+\ldots\right.
\end{gathered}
$$

hold. Substituting the last expansion in the representation of the integrand we define the residue in zero. Thus we have

$$
\left((\bar{E}-\bar{A})^{-1} \bar{B}\right) \bar{f}+\sum_{i=1}^{\infty} \frac{\bar{x}_{0}^{(k)} \bar{z}_{m_{k}}^{(k)}+\ldots+\bar{x}_{m_{k}}^{(k)} \bar{z}_{0}^{(k)}}{\lambda_{i}^{2}}=0
$$

We obtain the assertion about possibility of expansion of elements from range of operator $(\bar{E}-\bar{A})^{-1} \bar{B}$ on eigen and associated vectors of the operator $\bar{L}(\lambda)$. For the completing of the proof of this theorem we need in the statement of connections between the eigen and associated vectors of operators $\overline{(E}-\lambda(\bar{E}-\bar{A})^{-1} \bar{B}^{-1}$ and $L(\lambda)$.

The eigen and associated vectors of the operator $\bar{E}-\lambda(\bar{E}-\bar{A})^{-1} \bar{B}$ coincides with the eigen and associated vectors of the operator $\bar{E}-\bar{A}-\lambda \bar{B}$, correspondingly. It is not difficult to state that the first components of eigen and associated vector of the operator $\bar{E}-\bar{A}-\lambda \bar{B}$ coincide with the eigen and associated vectors of the equation (1), correspondingly.

If $\left\{\bar{x}_{i}=\left(x_{0, i}, x_{1, i}, \ldots, x_{n-1, i}\right)\right\}_{1}^{\infty}$ is the system of eigen and associated vectors of (5) then $\left\{x_{0, i}\right\}_{1}^{\infty}$ is the system of eigen and associated vectors of the operator $L(\lambda)$. Components $x_{k, i}(k=1,2, \ldots, n-1)$ of eigen and associated vectors of the system $\left\{\bar{x}_{i}=\left(x_{0, i}, x_{1, i}, \ldots, x_{n-1, i}\right)\right\}_{1}^{\infty}$ of the operator $\bar{L}(\lambda)$ are defined with the help of the formulas

$$
\begin{aligned}
& x_{k, i}=B^{k}\left(\lambda^{k} x_{0, i}+k \lambda^{k-1} x_{0, i-1}+\frac{k(k-1)}{2 !} \lambda^{k-2} x_{0, i-2}+\ldots\right. \\
& \left.+\frac{k(k-1) \ldots 1}{k !} x_{0,0}\right)
\end{aligned}
$$

where $\left\{x_{0, i}\right\}$ is the chain of eigen and associated vectors of the operator $L(\lambda)$. So the closure of the range $\left.R(\bar{E}-\bar{A})^{-1} \bar{B}\right)$ of the operator $\left((\bar{E}-\bar{A})^{-1} \bar{B}\right)$ coincides with the whole space $\bar{H}$, then the completeness of the eigen and associated vectors of the operator $(\bar{E}-\bar{A})^{-1} \bar{B}$ in the space $\bar{H}$ takes place. The last means the $n$-multiple completeness of eigen and associated vectors of the pencil $L(\lambda)$ in the Hilbert space $H$. Moreover, element from the range of the operator $\left((\bar{E}-\bar{A})^{-1} \bar{B}\right)$ is expanded on the system of the eigen and associated vectors of the operator $(\bar{E}-\bar{A})^{-1} \bar{B}$ in the space $\bar{H}$ Therefore we proved the possibility of summation of any of $n$ elements $f_{0}, f_{1}, \ldots, f_{n-1 ;}$ of the space $H$ on the systems $x_{k, i}(k=0,1,2, \ldots, n-1) \quad(12), \quad$ correspondingly, with the brackets and the same coefficients.

The theorem is proved. 


\section{Conclusion}

It is proved the convergence of $\mathrm{n}$ series on eigen and associated vectors of operator pencil of Keldysh in the Hilbert space.

\section{References}

[1] Keldysh M.V. About completeness of eigen functions of some class linear non-selfadjoint operators. Journal Success of Mathematical Science .1971, т.27, issue.4,p.15-47

[2] Dzhabarzadeh R.M. On expansions series on eigen and associated vectors of operator pencils Journal: Scientific notes of Azerb.State University,1964, №3,pp.75-81.

[3] Vizitei V.N., Markus A.S. On convergence of multiple expansions on the system of eigen and associated vectors of polynomial pencils /Mathematical collection,1965,т.66, №2, pp..287-320

[4] Gokhberg I.Ts., Kreyn M.Q. Introduction to the theory of linear non-selfadjoint operators in the Hilbert space.Moscow,1964, pp 1-433.

[5] Allakhverdiev J.E., Dzhabarzadeh R. M. // Spectral theory of operator pencil in the Hilbert space. ДАN of Azerbaijan, 2011, т.LXVII, № 4., pp.3-10.

[6] Allakhverdiev J.E.,Dzhabarzadeh R.M. On summation of multiple series on eigen and associated vectors of operator pencils by Abel's method. ДAN Az SSR, 1979,т.35, № 7,p 1923.

[7] Lidskii V.B.. About summation of the series on the general vectors of the non-selfadjoint operators. Proceeding of Moscow Scientific Society, t.11, 1962.

[8] Radzievskii Q.V.Multiple completeness of root vectors of Keldysh's operator pencil, disturbing by analytical operatorfunction.DAN USSR,ser.A,1976,,7,pp.597-600.

[9] Dzhabarzadeh R. M. To the spectral theory of polynomial pencils.Proceedinfg of Oryol State Universitety Russia ,2006,t.1,pp.161-165. 